\title{
Dysphagia in an elderly due to change in cervical spine curvature treated by manual therapy - A diagnostic and therapeutic challenge
}

\author{
Shyam Jungade ${ }^{1}$, Shraddha Jain ${ }^{2, *}$, Shalaka D. Khobragade ${ }^{3}$, Priyanka A. Telang ${ }^{4}$ \\ ${ }^{1}$ Associate Professor, ${ }^{2}$ Professor \& HOD, ${ }^{3,4}$ Resident, ${ }^{1,3,4}$ Dept. of Community Health Sciences, Ravi Nair Physiotherapy College, \\ Datta Meghe Institute of Medical Sciences, Deemed to be University, Sawangi, Wardha, Maharashtra, ${ }^{2}$ Dept. of \\ Otorhinolaryngology and Head and Neck Surgery, Jawahar Lal Nehru Medical College, Datta Meghe Institute of Medical \\ Sciences, Deemed to be University, Sawangi, Wardha, Maharashtra, India
}

*Corresponding Author:

Email: sjain_med@yahoo.co.in

\begin{abstract}
Dysphagia in elderly could be attributable to a number of etiological causes ranging from neurologic causes like stroke, malignancies, motility disorders, cervical osteophytic compression and changes in cervical spine curvature.

There are studies which have reported the occurrence of dysphagia in patients with cervical spine osteophytes. However, the loss of cervical lordosis as a cause of dysphagia has not received much attention and is primarily a diagnosis of exclusion. Most of the studies have focused on dysphagia following anterior cervical spine surgery.

This is probably first report of a case of dysphagia attributable to cervical spine curvature change due to neck muscle spasm, which was treated by manual therapy, in a 70 year old male, where all other known causes of dysphagia were excluded. We found improvement in angle of cervical lordosis between C-2 and C-7 vertebrae both by "Posterior tangent technique" and "Cobb Angle Method".
\end{abstract}

Keywords: Cervical spine, Lordosis, Dysphagia, Manual therapy, Elderly.

\section{Background}

Dysphagia in elderly is multifactorial. The causes can range from neurological causes like stroke, malignancies, cervical osteophytic compression ${ }^{1}$ and changes in cervical spine curvature. $^{2}$ The loss of cervical lordosis as a cause of dysphagia has not received much attention and is primarily a diagnosis of exclusion. Most of the studies have focused on dysphagia following anterior cervical spine surgery.

In order to restore cervical lordosis, few conservative methods of rehabilitating sagittal cervical alignment have been reported. Manual physical therapy is a specialized form of physical therapy delivered with the hands as opposed to a device or machine. In manual therapy, therapists use their hands to put pressure on fascia and muscle tissue in an attempt to correct the muscle spasm, muscle tension, and joint dysfunction.

Here, we report an interesting case of dysphagia in an elderly male, who presented to otolaryngology department, on the suspicion of a post-cricoid malignancy as flexible endoscope could not be negotiated, which was completely relieved by manual therapy for loss of cervical lordosis.

This is probably first report of a case of dysphagia attributable to cervical spine curvature change due to neck muscle spasm, which was treated by manual therapy, with resultant improvement in cervical lordosis.

\section{Case Description}

A 70 year old male presented to otolaryngology department with complaint of difficulty in swallowing, which was associated with pain. There was no history of dysphonia or dyspnea. There was a positive history of neck pain. Upper Gastro-intestinal flexible endoscopy had been done previously and scope could not be negotiated due to post cricoid narrowing. Rigid laryngeal videoendoscopy revealed pooling of saliva in both pyriform fossae with bilaterally congested arytenoids and normal cord mobility. Rigid oesophagoscopy did not reveal any mass or narrowing, however, scope could be introduced with difficulty due to change in cervical spine curvature. Barium swallow examination was normal.

There was trapezius spasm on neck examination. Lateral neck radiograph revealed mild straightening of cervical spine. The $\mathrm{C} 2-\mathrm{C} 7$ angle by "Posterior tangent measurement technique" (Fig. 1) and "The Cobb angle, (Fig. 2). were $52.47^{\circ}$ and $56.42^{\circ}$ respectively.

The patient was subjected to conservative treatment in the form of steroids and anti-inflammatory drugs. Physical intervention comprised of manual therapy in the form of myofascial release for the trapezius muscle and cervical spine mobilization along with isometric exercises for posterior neck muscles The manual therapy comprised of soft tissue mobilization (STM) in order to break up inelastic or fibrous muscle tissue (called 'myofascial adhesions') such as scar tissue from a previous injury, to move tissue fluids, and to relax muscle tension. This procedure in our case was applied to the fascia around trapezius and consisted of deep pressure. The area of greatest tissue restriction was localized through layer-bylayer assessment. Once identified, these restrictions could be mobilized with deep digital pressure of pre-determined intensity and rhythm. 


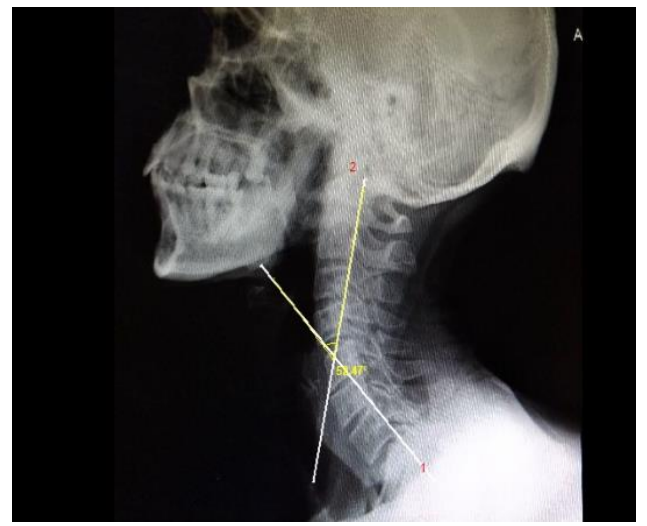

Fig. 1: Pre -treatment measurement of cervical lordosis angle between $\mathrm{C}-2$ and $\mathrm{C}-7$ by "posterior tangent technique"

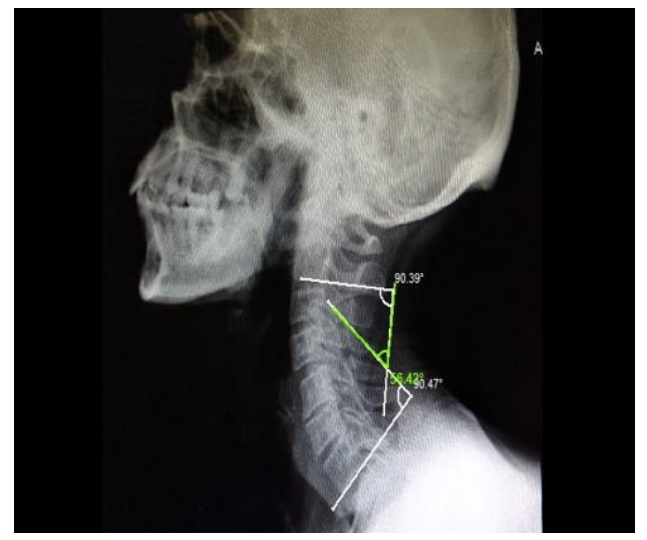

Fig. 2: Pre -treatment measurement of cervical lordosis angle between $\mathrm{C}-2$ and $\mathrm{C}-7$ by "Cobb angle technique"

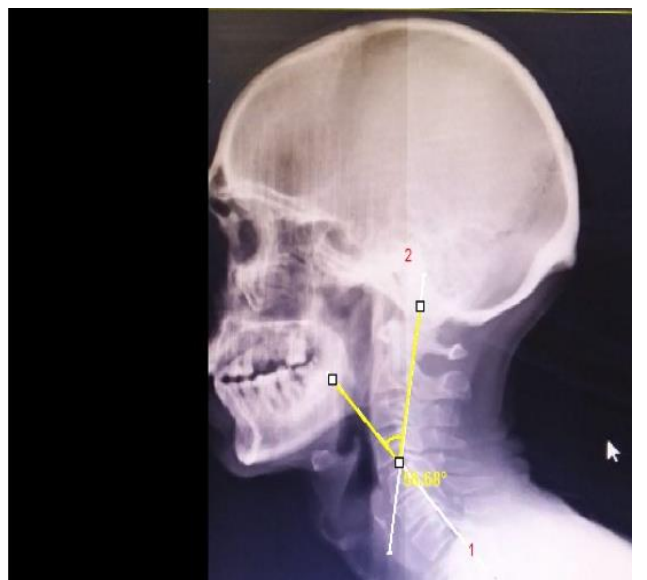

Fig. 3: Post -treatment measurement of cervical lordosis angle between $\mathrm{C}-2$ and $\mathrm{C}-7$ by "posterior tangent technique" showing improvement in cervical lordotic curvature

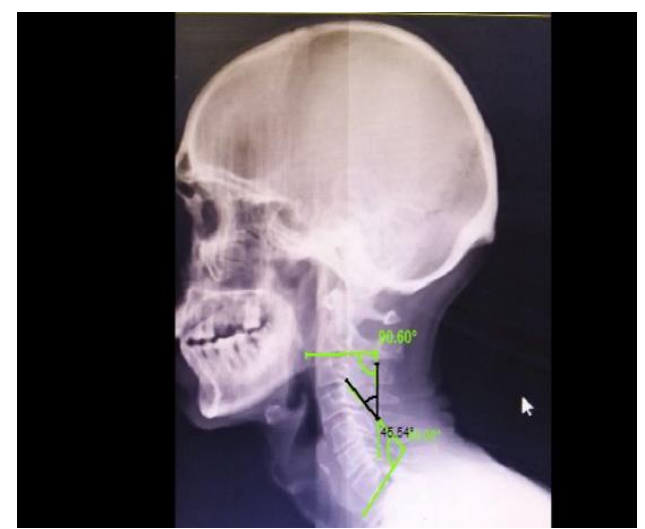

Fig. 4: Post -treatment measurement of cervical lordosis angle between $\mathrm{C}-2$ and $\mathrm{C}-7$ by "Cobb angle technique" showing improvement in cervical lordotic curvature

\section{Outcome}

Single session of treatment resulted in immediate relief of dysphagia and patient started taking solids orally. The post manual therapy lateral neck radiograph revealed restoration of cervical lordotic curvature and $\mathrm{C} 2$ $-\mathrm{C} 7$ angle by "Posterior tangent measurement technique" improved from $52.47^{\circ}$ to $56.68^{\circ}$ (Fig. 3) and "The Cobb angle, improved from $56.42^{\circ}$ to $45.54^{\circ}$ (Fig. 4). Patient had significant symptomatic relief till six months follow up.

\section{Discussion}

Cervical spine abnormalities as a cause of dysphagia, are an often neglected entity. Most of the cases of dysphagia due to pathology in cervical spine are attributable to anterior osteophytes. ${ }^{1}$ In our patient, decrease in cervical lordosis was attributable to spasm of neck muscles, as a cause of dysphagia. An association has been observed between deglutition and physiological lordosis of cervical spine. ${ }^{2}$ Mekata et al, $2013^{2}$ observed that the cervical spine moves to reduce physiological lordosis during deglutition and that the decrease of cervical motion in elderly population may have negative influence on swallowing. Others have also observed the Influence of neck postural changes on cervical spine motion and angle during swallowing, especially that of "retraction posture". ${ }^{3}$

Various authors have studied angles between second and seventh cervical vertebrae to define physiologic lordosis and also attempted to define conditions of any deviations from the normal. Nagata et al, 2011 ${ }^{4}$ evaluated the total alignment of the cervical spine with the $\mathrm{C} 2-7$ angle of spinal curvature by drawing a line from the posterior inferior corner of the $\mathrm{C} 2$ vertebral body down to the posterior inferior corner of the $\mathrm{C} 7$ vertebral body. Lordosis is said to be present if all intervening vertebral bodies are anterior to this line. If at least one vertebral body is traversed by the line, cervical alignment is judged to be straight and if the intervening vertebral bodies are posterior to the line, kyphosis is 
present. They found C2-7 angle of spinal total alignment ranged from 0 to 55.8 degrees with an average of 17.6 degrees. ${ }^{4}$ The two commonly measured angles for cervical lordosis on lateral radiograph of neck are "Posterior tangent measurement technique" and "The Cobb angle, the angle determined by the intersection of the perpendiculars". 5

The clinical significance of change in physiologic cervical lordosis has been studied by various authors. McAviney et al., $2005^{6}$ found a statistically significant association between cervical pain and lordosis of $<20^{\circ}$ and a "clinically normal" range for cervical lordosis of $31^{\circ}-40^{\circ}$. They, suggested maintenance of a lordosis in the range of $31^{\circ}-40^{\circ}$ could be a clinical goal for chiropractic treatment.

In our study, manual therapy resulted in immediate improvement of dysphagia by restoration of cervical spine lordosis. However, other studies have reported variable results of spinal manipulation in restoration of cervical lordosis. Harrison DE et al, $2003^{7}$ employed cervical manipulation and a cervical extensioncompression traction (vertical weight applied to the subject's forehead in the sitting position with a transverse load at the area of kyphosis) for restoration of cervical lordosis. The parameters of improvement in their study were Cobb angle at C2-C7 (mean improvement of -13.6 degrees), and the angle of intersection of the posterior tangents at $\mathrm{C} 2-\mathrm{C} 7$ (mean improvement of 17.9 degrees). Our case also showed improvement of -10.9 degrees in "Cobb angle" at C2-C7 and improvement of 4.21 degree in the "angle of intersection of the posterior tangents" at $\mathrm{C} 2-\mathrm{C} 7$. We observed greater improvement in the Cobb angle in this case. Moustafa et al. ${ }^{8}$ devised methods for the restoration of cervical lordosis as a means to improve neck pain and related disability. Another study by Shilton et al., 2015, ${ }^{9}$ found no effect of spinal manipulation on cervical lordosis after 4 weeks of cervical spinal manipulation therapy.

Presence of weakened or shortened muscles as a cause of change in body musculoskeletal biomechanics is an indication to use soft tissue techniques of manual therapy. Once pain has been reduced and joint mobility improved with the application of manual therapy, the patient can regain normal movement patterns and restore maximal function. ${ }^{10}$

\section{Conclusion}

1. We conclude that manual therapy holds promise in treatment of dysphagia in elderly due to straightening of cervical spine or decrease in lordotic curvature, after excluding other causes.
2. There is need to define critical angles of cervical lordosis in the causation of dysphagia.

3. Also research is needed in identification of various mechanisms underlying improvement in function by manual therapy.

Funding: No funding sources.

Conflict of interest: None declared.

\section{References}

1. Strasser G, Schima W, Schober E et al. Cervical osteophytes impinging on the pharynx: importance of size and concurrent disorders for development of aspiration. AJR Am J Roentgenol 2000;174:449-53.

2. Mekata K, Takigawa T, Matsubayashi J, et al. Cervical spine motion during swallowing. Eur Spine $J$ 2013;22:2558-63.

3. Kim JY, Hong JT, Oh JS, Jain A, Kim IS, Lim SH, Kim JS. Influence of neck postural changes on cervical spine motion and angle during swallowing. Med (Baltimore) 2017;96(45):e8566.

4. Nagata T, Takami T, Yamagata T, Uda T, Naito K, Ohata K. Significant relationship between local angle at fused segments and C2-7 angle: Average duration of longer than 20 years after anterior cervical discectomy and fusion. J Craniovertebr Junction Spine 2011;2(2):62-6.

5. Lippa L, Lippa L, Cacciola F. Loss of cervical lordosis: What is the prognosis? J Craniovertebr Junction Spine 2017;8(1):9-14.

6. McAviney J, Schulz D, Bock R, Harrison DE, Holland B. Determining the relationship between cervical lordosis and neck complaints. J Manipulative Physiol Ther 2005;28:187-93.

7. Harrison DE, Harrison DD, Betz JJ, Janik TJ, Holland B, Colloca CJ, Haas JW. Increasing the cervical lordosis with chiropractic biophysics seated combined extensioncompression and transverse load cervical traction with cervical manipulation: nonrandomized clinical control trial. J Manipulative Physiol Ther 2003;26(3):139-51.

8. Moustafa IM, Diab AA, Harrison DE. The effect of normalizing the sagittal cervical configuration on dizziness, neck pain, and cervicocephalic kinesthetic sensibility: A 1-year randomized controlled study. Eur J Phys Rehabil Med 2017;53(1):57-71.

9. Shilton M, Branney J, de Vries BP, Breen AC. Does cervical lordosis change after spinal manipulation for non-specific neck pain? A prospective cohort study. Chiropr Man Therap 2015;23:33.

10. Bialosky JE, Bishop MD, Price DD, Robinson ME, George SZ. The mechanisms of manual therapy in the treatment of musculoskeletal pain: a comprehensive model. Man Ther 2009;14(5):531-38.

How to cite the article: Jungade S., Jain S., Khobragade S., Telang P. Dysphagia in an elderly due to change in cervical spine curvature treated by manual therapy - A diagnostic and therapeutic challenge. Indian J Anat Surg Head Neck Brain 2018;4(3):91-93. 